\title{
Comparison of Proposed Modified and Original Sequential Organ Failure Assessment Scores in Predicting ICU Mortality: A Prospective, Observational, Follow-Up Study
}

\author{
Afshin Gholipour Baradari, ${ }^{1}$ Hassan Sharifi, ${ }^{2}$ Abolfazl Firouzian, ${ }^{1}$ \\ Maryam Daneshiyan, ${ }^{1}$ Mohsen Aarabi, ${ }^{3}$ Yaser Talebiyan Kiakolaye, ${ }^{4}$ \\ Seyed Mahmood Nouraei, ${ }^{5}$ Alieh Zamani Kiasari, ${ }^{1}$ Mohammad Reza Habibi, ${ }^{1}$ \\ Amir Emami Zeydi, ${ }^{6}$ and Faegheh Sadeghi ${ }^{7}$ \\ ${ }^{1}$ Department of Anesthesiology, Faculty of Medicine, Mazandaran University of Medical Sciences, Sari, Iran \\ ${ }^{2}$ Department of Medical-Surgical Nursing, School of Nursing and Midwifery, Iranshahr University of Medical Sciences, \\ Iranshahr, Iran \\ ${ }^{3}$ Department of Epidemiology, Faculty of Medicine, Mazandaran University of Medical Sciences, Sari, Iran \\ ${ }^{4}$ Critical Care Nursing, Cancer Institute, Tehran University of Medical Sciences, Tehran, Iran \\ ${ }^{5}$ Department of Cardiac Surgery, Faculty of Medicine, Mazandaran University of Medical Sciences, Sari, Iran \\ ${ }^{6}$ Department of Medical-Surgical Nursing, Faculty of Nursing and Midwifery, Mazandaran University of Medical Sciences, \\ Sari, Iran \\ ${ }^{7}$ Faculty of Medicine, Mazandaran University of Medical Sciences, Sari, Iran
}

Correspondence should be addressed to Hassan Sharifi; sharifi9879@hotmail.com

Received 3 September 2016; Revised 1 November 2016; Accepted 8 November 2016

Academic Editor: Robert Boots

Copyright (C) 2016 Afshin Gholipour Baradari et al. This is an open access article distributed under the Creative Commons Attribution License, which permits unrestricted use, distribution, and reproduction in any medium, provided the original work is properly cited.

\begin{abstract}
Background. The sequential organ failure assessment (SOFA) score has been recommended to triage critically ill patients in the intensive care unit (ICU). This study aimed to compare the performance of our proposed MSOFA and original SOFA scores in predicting ICU mortality. Methods. This prospective observational study was conducted on 250 patients admitted to the ICU. Both tools scores were calculated at the beginning, 24 hours of ICU admission, and 48 hours of ICU admission. Diagnostic odds ratio and receiver operating characteristic (ROC) curve were used to compare the two scores. Results. MSOFA and SOFA predicted mortality similarly with an area under the ROC curve of $0.837,0.992$, and 0.977 for MSOFA 1, MSOFA 2, and MSOFA 3, respectively, and $0.857,0.988$, and 0.988 for SOFA 1 , SOFA 2 , and SOFA 3, respectively. The sensitivity and specificity of MSOFA 1 in cut-off point 8 were $82.9 \%$ and $68.4 \%$, respectively, MSOFA 2 in cut-off point 9.5 were $94.7 \%$ and $97.1 \%$, respectively, and MSOFA 3 in cut-off point of 9.3 were $97.4 \%$ and $93.1 \%$, respectively. There was a significant positive correlation between the MSOFA 1 and the SOFA 1 $(r: 0.942), 24$ hours $(r: 0.972)$, and 48 hours $(r: 0.960)$. Conclusion. The proposed MSOFA and the SOFA scores had high diagnostic accuracy, sensitivity, and specificity for predicting mortality.
\end{abstract}

\section{Introduction}

Organ dysfunction and/or failure is one of the main causes of mortality and morbidity in patients hospitalized in the intensive care unit (ICU) $[1,2]$. Prediction of mortality and morbidity in the ICUs can enhance clinical decision-making
[3], facilitate classifying the critically ill patients according to their health status [4], and improve the quality of provided services for critically ill patients $[5,6]$.

Several methods for predicting critically patient outcomes are available, including sequential organ failure assessment (SOFA) [7], Acute Physiology and Chronic Health 
Evaluation (APACHE) [8], Simplified Acute Physiology Score (SAPS), and the Multiple Organ Dysfunction Score (MODS) [9]. The SOFA is one of the useful tools for evaluating organ dysfunction and failure as well as predicting mortality rate in critically ill patients [9].

Previously, the accuracy of SOFA has been determined by several studies and predictive power of this tool has been proven in the critically ill patients with various diseases hospitalized in the ICU $[1,7,10]$. Some studies have reported that the discriminatory power and calibration of the SOFA is higher than the other tools such as Acute Physiology and Chronic Health Evaluation (APACHE) and Simplified Acute Physiology Score (SAPS) [11, 12]. In addition, the ability to review data on a daily basis or every 24 hours in patients is another advantages, whereas most of the other tools are applicable only in the first 24 hours $[6,10]$. However, some variables of the SOFA score are expensive or unavailable in some hospitals. The cost of performing SOFA is high and requires specific laboratory equipment that is scarce in some hospitals $[13,14]$. To reduce their costs, many hospitals use the traditional methods for evaluating the patient's outcomes such as ICU mortality and morbidity [13]. For this reason, some researchers designed and developed the modified version of SOFA (MSOFA) score as a cost-effective alternative with easy application for predicting mortality in ICU $[14,15]$.

In the present study, we have proposed another slightly modified version of original SOFA (proposed MSOFA) according to its recognized advantages and disadvantages. Therefore, our study aimed to compare the performance of our proposed MSOFA and original SOFA scoring systems in predicting ICU mortality.

\section{Methods}

This prospective observational study was conducted in the ICU of 600-bed Imam Khomeini hospital, Mazandaran University of Medical Sciences, Sari, Iran, during December 2014 to March 2015. Approval for the project was obtained from the University Ethics Committee.

2.1. Eligibility Criteria. All patients older than 18 years with more than 24 hours hospitalization were included. Because of false increased ICU mortality rate, all patients with limits on life-sustaining interventions were excluded, for example, patients who died in less than 24 hours of ICU admission or received CPR before ICU admission. Patients were followed up until ICU discharge in order to document their survival status. ICU nursing staff and physicians were trained theoretically and practically how to calculate and document the proposed modified and original SOFA scores.

2.2. Data Collection. Data was collected by research assistances (ICU nursing staff and physicians) in three stages at eight o'clock every morning. The worst physiological score of each organ was calculated using original SOFA and MSOFA at three time series (from number 1 to 3 ): at the beginning, 24 hours after ICU admission, and then 48 hours after ICU admission. The key variables in this study were main causes of hospitalization in the ICU, length of stay in ICU and hospital outcome, proposed MSOFA and original SOFA scores, and chart of recorded scores for the organs.

2.3. Tools. The original SOFA score assesses the severity of disease and evaluates the function of 6 body vital organs on a daily basis [7]. The criteria are as follows: respiratory system with the $\mathrm{PO}_{2} / \mathrm{FiO}_{2}$; cardiovascular system with mean arterial pressure (MAP) and the amount of drug needed to increase blood pressure; coagulation system by measuring platelet counts; liver system by measuring total bilirubin level; nervous system by measuring the Glasgow Coma scale; and renal system with urinary output and creatinine levels. Each organ scores from zero to four, and the total score for all organs calculated from zero to 24 . Scores 1-2 determine organ dysfunction while scores 3-4 represent organ failure. The scores were evaluated every 24 hours. If each variable were repeated several times during 24 hour, the worst score would be recorded. After scoring each organ, the total SOFA score was calculated for all patients.

Our proposed MSOFA is derived from the original SOFA score and it has some advantages. MSOFA does not require specific laboratory equipment, is calculated easily on the patient bedside, and is available for daily reassessment. It has six different criteria for each organ, including the respiratory, coagulation, cardiovascular, liver, nervous, and renal. In the following, we describe the modifications. (1) The respiratory system was evaluated by $\mathrm{SPO}_{2} / \mathrm{FiO}_{2}$ criteria. $\mathrm{SPO}_{2}$ was obtained using pulse oximetry, and $\mathrm{FiO}_{2}$ calculated by the percentage of oxygen adjusted in the ventilator or the percentage of oxygen delivered to the patient by airway. $\mathrm{SPO}_{2} / \mathrm{FiO}_{2}$ is shown as a decimal number in the denominator; for example, $\mathrm{FiO}_{2}=55 \%$ is written as 0.55 . (2) The coagulation system was evaluated with clinical assessment with a score of two for petechia, purpura, and ecchymosis, and a score of four for spontaneous bleeding. (3) The cardiovascular system was assessed by mean atrial pressure (MAP) and the need for vasoactive drugs. If MAP is less than $70 \mathrm{mmHg}$, vasoactive drugs such as dopamine, dobutamine, epinephrine, and norepinephrine would be prescribed in accordance with hospital policy charting system. Scoring is done based on the required dose of the drug for preserving MAP more than $70 \mathrm{mmHg}$. (4) The function of liver system was scored by clinical assessment of jaundice with a score of two for jaundice in the sclera and a score of four for jaundice in the skin. (5) The central nervous system was assessed by GCS level. (6) The renal system was assessed by urinary output level based on $\mathrm{cc} / \mathrm{kg} / \mathrm{h}$.

2.4. Study Outcome. The main outcome of the present study was evaluating the sensitivity, specificity, positive, and negative predictive values of our proposed modified SOFA for predicting ICU mortality and disability of the patient until discharge from the ICU and compare these values with the original SOFA scores.

2.5. Data Analysis. All statistical analysis was performed using the Statistical Program for Social Sciences (SPSS ver. 18) software. We used descriptive statistics to determine the characteristics of the sample and to describe the study 
TABLE 1: Patients characteristics.

\begin{tabular}{|c|c|c|}
\hline Properties & Categories & Values \\
\hline \multirow{2}{*}{ Age (year) } & $\begin{array}{c}\text { Male } \\
154(61.6 \%)\end{array}$ & $\begin{array}{c}44.39 \pm 19.83 \\
\text { (ranged 18-48) }\end{array}$ \\
\hline & $\begin{array}{c}\text { Female } \\
96(38.4 \%) \\
\end{array}$ & $\begin{array}{c}48.50 \pm 18.69 \\
\text { (ranged 18-82) }\end{array}$ \\
\hline \multirow{2}{*}{$\begin{array}{l}\text { Cause of } \\
\text { hospitalization }\end{array}$} & Traumatic & $101(40.4 \%)$ \\
\hline & Nontraumatic & $140(59.4 \%)$ \\
\hline \multirow{5}{*}{ History } & Underlying disease & $145(58 \%)$ \\
\hline & $\begin{array}{l}\text { Prior history of } \\
\text { hospitalization }\end{array}$ & $33(13.2 \%)$ \\
\hline & $\begin{array}{l}\text { Required } \\
\text { intubation }\end{array}$ & $164(65.6 \%)$ \\
\hline & $\begin{array}{l}\text { Required } \\
\text { tracheostomy }\end{array}$ & $8(3.2 \%)$ \\
\hline & $\begin{array}{l}\text { Required } \\
\text { reintubation }\end{array}$ & $4(1.6 \%)$ \\
\hline \multirow{6}{*}{$\begin{array}{l}\text { Patients morbid- } \\
\text { ity/mortality } \\
\text { outcomes }\end{array}$} & Brain death & $2(0.8 \%)$ \\
\hline & $\begin{array}{l}\text { Transfer to the } \\
\text { other centers }\end{array}$ & $7(2.8 \%)$ \\
\hline & Vegetative state & $2(0.8 \%)$ \\
\hline & $\begin{array}{l}\text { Quadriplegia in the } \\
\text { ICU }\end{array}$ & $1(0.4 \%)$ \\
\hline & $\begin{array}{l}\text { Quadriplegia out } \\
\text { of the ICU }\end{array}$ & $2(0.8 \%)$ \\
\hline & Death & $78(31.2 \%)$ \\
\hline
\end{tabular}

variables. Data were presented as mean $+\mathrm{SD}$, when indicated. Pearson correlation coefficients were calculated to quantify associations between SOFA and MSOFA. Diagnostic odds ratio and ROC curve were used to compare both modified and original scores for sensitivity, specificity, positive, and negative predictive values for the prediction of mortality and disability. The ability of the models for predicting ICU mortality was determined by examining their discrimination, which is the ability of a model to distinguish between a patient who will live and one who will die. Discrimination and/or accuracy was measured by examining the area under the receiver operating characteristics curve (AUC). An area of 1 represents a perfect test; an area of .5 represents a worthless test. The curves were constructed by computing the sensitivity and specificity of increasing numbers of clinical findings (from 0 to 1 ) in predicting mortality. The model has good discrimination when the AUC is more than 0.8 . The difference in the AUC was analyzed by the $z$ statistic. The significance level was set at 0.05 , and $95 \%$ confidence interval was calculated for all of cases.

\section{Results}

3.1. Patients Characteristics. A total of 250 patients were included (mean age $45.97 \pm 19.46$ years, ranged 18-82). There was no statistically difference between men and women age $(P=0.106)$. Table 1 depicts some characteristics of patients.

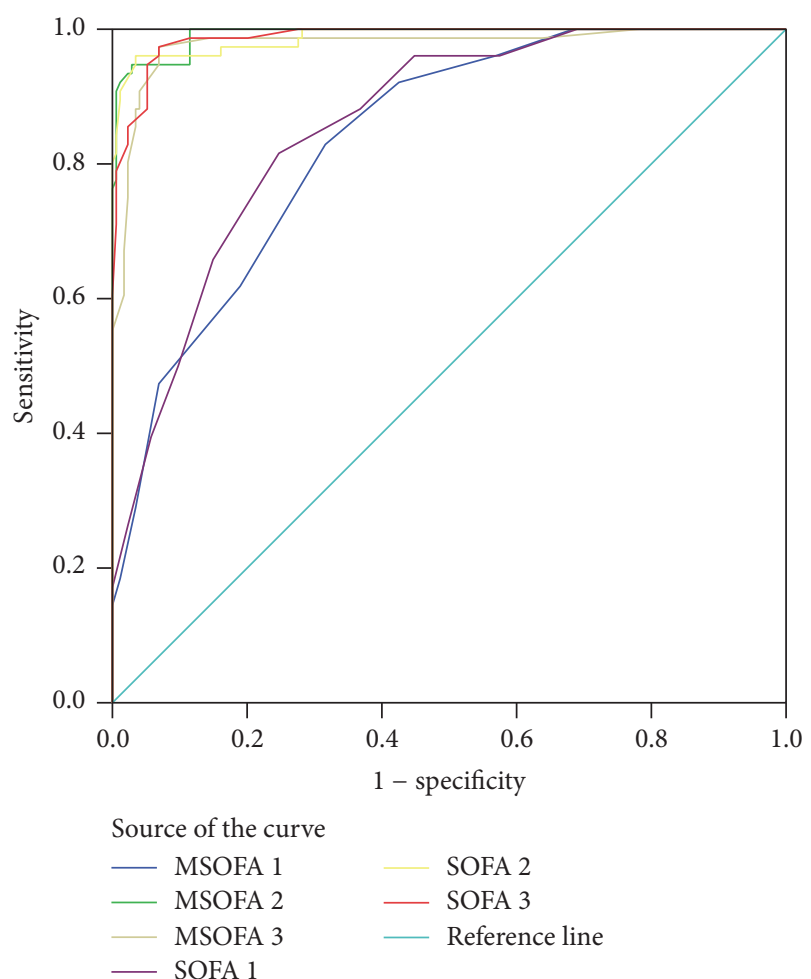

FIGURE 1: Comparing the predictive power SOFA 1, SOFA 2, and SOFA 3 and MSOFA 1, MSOFA 2, and MSOFA 3, in predicting mortality using ROC curve.

The causes of death were cardiac arrest, intracranial hemorrhage ( $\mathrm{ICH}$ ), and malignancy (cancer).

Table 2 compares some of the main characteristics of the sample between deceased and survived patients. The mean age of deceased patients was significantly higher than those survived. Most of the deceased patients were females. The duration of ventilation and hospitalization in the ICU were significantly higher in deceased patients. The scores of MSOFA 1, MSOFA 2, and MSOFA 3 as well as SOFA 1, SOFA 2, and SOFA 3 were significantly higher in the deceased patients.

3.2. Study Outcomes. There was a significant positive correlation between the MSOFA 1 and SOFA $1(r: 0.942, P<$ $0.0001)$, MSOFA 2 and SOFA $2(r: 0.972, P<0.0001)$, and also MSOFA 3 and SOFA $3(r: 0.960, P<0.0001)$. The performance of MSOFA and SOFA score is evaluated in Figure 1. Interpretation of the area under the ROC curve (AUC) showed that the performance of MSOFA 1 score with cut-off point of eight was AUC 0.837 (95\% CI: 0.788-0.887). In predicting mortality, the sensitivity, specificity, positive predictive value (PPV), and negative predictive value (NPV) were $82.9 \%, 68.4 \%, 53.4 \%$, and $90.2 \%$, respectively.

The MSOFA 2 with the cut-off point of 9.5 (AUC: 0.992 , 95\% CI: 0.985-0.999; $P<0.0001$ ) had 94.7\%, 97.1\%, 93.5\%, and $97.7 \%$ sensitivity, specificity, positive predictive value (PPV), and negative predictive value (NPV) in predicting mortality, respectively. Table 3 compares the area under the curve (AUC) for each tool with 95\% CI. 
TABLE 2: Comparison of demographic data, clinical findings, and SOFA-MSOFA scores.

\begin{tabular}{|c|c|c|c|}
\hline Factors examined & $\begin{array}{c}\text { Deceased patients } \\
n=78(31.2 \%)\end{array}$ & $\begin{array}{c}\text { Survived patients } \\
n=172(68.8 \%)\end{array}$ & $P$ value \\
\hline Age $(M$ of years $\pm \mathrm{SD})$ & $51.29 \pm 17.72$ & $43.55 \pm 19.78$ & 0.003 \\
\hline \multicolumn{4}{|l|}{ Gender as $n(\%)$} \\
\hline Male & $41(26.62 \%)$ & $113(73.37 \%)$ & \multirow{2}{*}{0.048} \\
\hline Female & $37(38.54 \%)$ & $59(61.46 \%)$ & \\
\hline Duration of intubation (days) & $1.05 \pm 0.32$ & $1.03 \pm 0.23$ & 0.691 \\
\hline Duration of ventilation (days) & $9.22 \pm 9.10$ & $4.62 \pm 6.12$ & 0.048 \\
\hline Length of ICU stay (days) & $7.96 \pm 6.17$ & $4.87 \pm 4.86$ & $<0.0001$ \\
\hline Length of hospital stay (days) & $9.24 \pm 7.51$ & $8.50 \pm 6.09$ & 0.41 \\
\hline \multicolumn{4}{|l|}{ MSOFA } \\
\hline 1 & $9.93 \pm 3.17$ & $5.98 \pm 2.62$ & $<0.0001$ \\
\hline 2 & $14.67 \pm 3.88$ & $5.19 \pm 2.49$ & $<0.0001$ \\
\hline 3 & $14.26 \pm 3.88$ & $5.54 \pm 2.55$ & $<0.0001$ \\
\hline \multicolumn{4}{|l|}{ SOFA } \\
\hline 1 & $10.92 \pm 3.06$ & $6.31 \pm 2.94$ & $<0.0001$ \\
\hline 2 & $15.71 \pm 3.60$ & $5.51 \pm 2.83$ & $<0.0001$ \\
\hline 3 & $15.27 \pm 3.30$ & $5.98 \pm 2.85$ & $<0.0001$ \\
\hline
\end{tabular}

TABLE 3: Comparison of predictive power of SOFA 1, SOFA 2, and SOFA 3 and MSOFA 1, MSOFA 2, and MSOFA 3, in predicting mortality.

\begin{tabular}{ccccc}
\hline Tools & \multirow{2}{*}{ AUC $^{*}$} & \multicolumn{2}{c}{ Confidence interval of 95\% } & P value \\
& & Lower bound & Upper bound & \\
\hline MSOFA & & & & \\
1 & 0.837 & 0.788 & 0.887 & $<0.0001$ \\
2 & 0.992 & 0.985 & 0.999 & $<0.0001$ \\
3 & 0.977 & 0.957 & 0.988 & $<0.0001$ \\
SOFA & & & & \\
1 & 0.857 & 0.811 & 0.904 & $<0.0001$ \\
2 & 0.988 & 0.977 & 1.000 & $<0.0001$ \\
3 & 0.988 & 0.978 & 0.997 & $<0.0001$ \\
\hline
\end{tabular}

*AUC: area under curve.

\section{Discussion}

The main purpose of the present study was to compare the proposed MSOFA and original SOFA in predicting mortality rate of critically ill patients admitted to the ICU. Our results showed that both tools predict mortality with high accuracy. In the present study, the original SOFA and MSOFA scores predicted mortality as an excellent tool at the beginning, 24 hours after ICU admission, and 48 hours after ICU admission. We also found that proposed MSOFA 2 had a higher accuracy than other times. The ROC analysis and the area under the curve (AUC) were used to differentiate the power of a tool for distinguishing between survived and deceased patients. Based on the evidence AUC $\geq 0.7$ shows acceptable tool and AUC $\geq 0.9$ indicates excellent tool. Several studies have compared SOFA. In consistence with our results, Grissom et al. compared the modified SOFA
$\left(\mathrm{SPO}_{2} / \mathrm{FiO}_{2}\right.$ ratio instead of $\mathrm{PaO}_{2} / \mathrm{FiO}_{2}$ and jaundice in the sclera and body instead of measuring bilirubin and removal of platelets) with the original SOFA one. In their study, on the first day MSOFA and SOFA scores predicted mortality with AUC of 0.83 and AUC of 0.84 and on the third day with AUC of 0.78 and 0.79 , respectively. They concluded that MSOFA could calculate mortality like original SOFA [16]. In another study, Oda et al. examined the MSOFA power to predict early mortality after Ventricular Assist Devices (VAD) placement. The MSOFA showed a good discrimination to determine the mortality risk of organ failure on the first 7 day with the maximum AUC of 0.99 (95\% CI: 0.96-1.0). The MSOFA score more than 11 for detecting mortality had sensitivity of $92.9 \%$ and specificity of $96.7 \%$ [17].

In our study, the highest sensitivity $(94.7 \%)$ and specificity (97.1\%) of the MSOFA were on the second day (48 hr.) at the cut-off point of 9.5 to predict mortality. In addition, on the third day MSOFA ( $72 \mathrm{hr}$.) in the cut-off point of 9.3 showed sensitivity of $97.4 \%$ and specificity of $93.1 \%$ for predicting mortality. Similar to our results, Oda et al. concluded that the MSOFA has high accuracy to predict perioperative mortality [17]. Apart from the predicting mortality in the ICUs, SOFA has been used in several conditions for predicting morbidity. For example, Oliveira-Neto et al. evaluated the power of SOFA score to predict maternal morbidity and found that SOFA score could effectively evaluate the severity and estimating prognosis of morbidity [18]. In another study, Lin et al. suggested the modified model of SOFA for clinical assessment of patients with acute renal failure who require kidney transplantation [19]. Halim et al. compared the APACHE II, SOFA, and MSOFA to predict mortality in the ICU patients undergoing surgery. In consistence with our results, the mean scores of SOFA and MSOFA were significantly higher in deceased patients. They reported that the predictive power 
of the APACHE II was lower (AUC: 0.69; $P<0.001$ ) than the predictive powers for the SOFA (AUC: $0.73 ; P<$ 0.001 ) and the initial MSOFA (AUC: $0.75 ; P<0.001$ ). Their results suggested that the mean and maximum SOFA and MSOFA scores are better indicators for predicting mortality in operated patients admitted to the ICU [14].

\section{Limitations}

When interpreting the results, the following study limitations have to be taken into account. First, a single center with partially small sample was used to compare the predictability of tools which places limitations on the quality of ICU care and external applicability. Therefore, a multicenter study would have given better external validity. The findings of our study need to be validated in a future prospective study. Second, we used a nonstandardized assessment of clinical parameters such as jaundice.

\section{Conclusion}

This study showed the excellent performance of the total MSOFA score in predicting patients mortality admitted in the ICU. The results of the present study demonstrated a strong positive correlation between the scores achieved from the original SOFA and the proposed MSOFA at the 24 hours, 48 hours, and 72 hours after ICU admission, and both tools are associated with high sensitivity and specificity for predicting mortality. Our proposed MSOFA due to lower cost and greater ease of application can be a proper alternative to the original SOFA. Therefore, we offer MSOFA as a more convenient and usable tool, particularly in the regions with limited resource.

\section{Competing Interests}

The authors declare that there is no conflict of interests regarding the publication of this paper.

\section{References}

[1] E. H. T. Anami, C. M. C. Grion, L. T. Q. Cardoso et al., "Serial evaluation of SOFA score in a Brazilian teaching hospital," Intensive \& Critical Care Nursing, vol. 26, no. 2, pp. 75-82, 2010.

[2] J.-R. Le Gall, "The use of severity scores in the intensive care unit," Intensive Care Medicine, vol. 31, no. 12, pp. 1618-1623, 2005.

[3] M. T. Keegan, O. Gajic, and B. Afessa, "Severity of illness scoring systems in the intensive care unit," Critical Care Medicine, vol. 39, no. 1, pp. 163-169, 2011.

[4] B. Afessa, O. Gajic, and M. T. Keegan, "Severity of illness and organ failure assessment in adult intensive care units," Critical Care Clinics, vol. 23, no. 3, pp. 639-658, 2007.

[5] V. D. Mayr, M. W. Dünser, V. Greil et al., "Causes of death and determinants of outcome in critically ill patients," Critical Care, vol. 10, no. 6, article R154, 2006.

[6] E. Cholongitas, M. Senzolo, D. Patch, S. Shaw, C. Hui, and A. K. Burroughs, "Review article: Scoring systems for assessing prognosis in critically ill adult cirrhotics," Alimentary Pharmacology \& Therapeutics, vol. 24, no. 3, pp. 453-464, 2006.
[7] J.-L. Vincent, A. de Mendonça, F. Cantraine et al., "Use of the SOFA score to assess the incidence of organ dysfunction/failure in intensive care units: results of a multicenter, prospective study," Critical Care Medicine, vol. 26, no. 11, pp. 1793-1800, 1998.

[8] W. A. Knaus, J. E. Zimmerman, D. P. Wagner, E. A. Draper, and D. E. Lawrence, "APACHE-acute physiology and chronic health evaluation: a physiologically based classification system," Critical Care Medicine, vol. 9, no. 8, pp. 591-597, 1981.

[9] J. C. Marshall, D. J. Cook, N. V. Christou, G. R. Bernard, C. L. Sprung, and W. J. Sibbald, "Multiple organ dysfunction score: a reliable descriptor of a complex clinical outcome," Critical Care Medicine, vol. 23, no. 10, pp. 1638-1652, 1995.

[10] S. P. Acharya, B. Pradhan, and M. N. Marhatta, "Application of "the Sequential Organ Failure Assessment (SOFA) score" in predicting outcome in ICU patients with SIRS," Kathmandu University Medical Journal, vol. 5, no. 20, pp. 475-483, 2007.

[11] R. P. Moreno, P. G. H. Metnitz, E. Almeida et al., "SAPS 3-from evaluation of the patient to evaluation of the intensive care unit. Part 2: development of a prognostic model for hospital mortality at ICU admission," Intensive Care Medicine, vol. 31, no. 10, pp. $1345-1355,2005$

[12] X. Castella, A. Artigas, J. Bion et al., "A comparison of severity of illness scoring systems for intensive care unit patients: results of a multicenter, multinational study," Critical Care Medicine, vol. 23, no. 8, pp. 1327-1335, 1995.

[13] L. Minne, A. Abu-Hanna, and E. de Jonge, "Evaluation of SOFAbased models for predicting mortality in the ICU: a systematic review," Critical Care, vol. 12, no. 6, article no. R161, 2008.

[14] D. A. Halim, T. W. Murni, and I. S. Redjeki, "Comparison of Apache II, SOFA, and modified SOFA scores in predicting mortality of surgical patients in intensive care unit at Dr. Hasan Sadikin General Hospital," Critical Care and Shock, vol. 12, no. 4, pp. 157-169, 2009.

[15] Q. Bao, B. Wang, L. Yu, H. Weng, J. Ge, and L. Li, "A modified prognostic score for critically ill patients with cirrhosis: an observational study," Journal of Gastroenterology and Hepatology, vol. 31, no. 2, pp. 450-458, 2016.

[16] C. K. Grissom, S. M. Brown, K. G. Kuttler et al., "A modified sequential organ failure assessment score for critical care triage," Disaster Medicine and Public Health Preparedness, vol. 4, no. 4, pp. 277-284, 2010.

[17] S. Oda, H. Hirasawa, T. Sugai et al., "Comparison of Sepsisrelated Organ Failure Assessment (SOFA) score and CIS (cellular injury score) for scoring of severity for patients with multiple organ dysfunction syndrome (MODS)," Intensive Care Medicine, vol. 26, no. 12, pp. 1786-1793, 2000.

[18] A. Oliveira-Neto, M. A. Parpinelli, J. G. Cecatti, J. P. Souza, and M. H. Sousa, "Sequential organ failure assessment score for evaluating organ failure and outcome of severe maternal morbidity in obstetric intensive care," The Scientific World Journal, vol. 2012, Article ID 172145, 8 pages, 2012.

[19] Y.-F. Lin, W.-J. Ko, V.-C. Wu et al., "A modified sequential organ failure assessment score to predict hospital mortality of postoperative acute renal failure patients requiring renal replacement therapy," Blood Purification, vol. 26, no. 6, pp. 547554, 2008. 


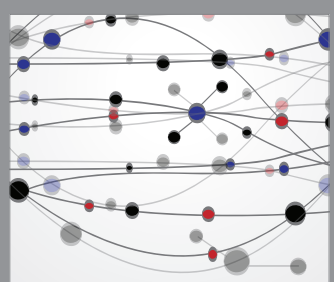

The Scientific World Journal
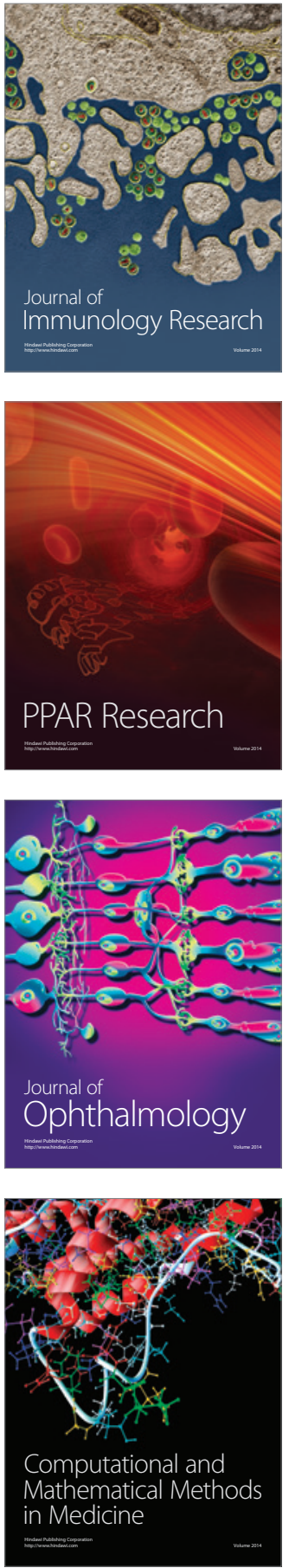

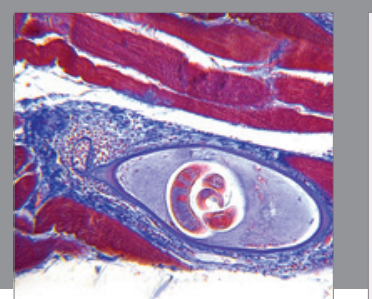

Gastroenterology Research and Practice

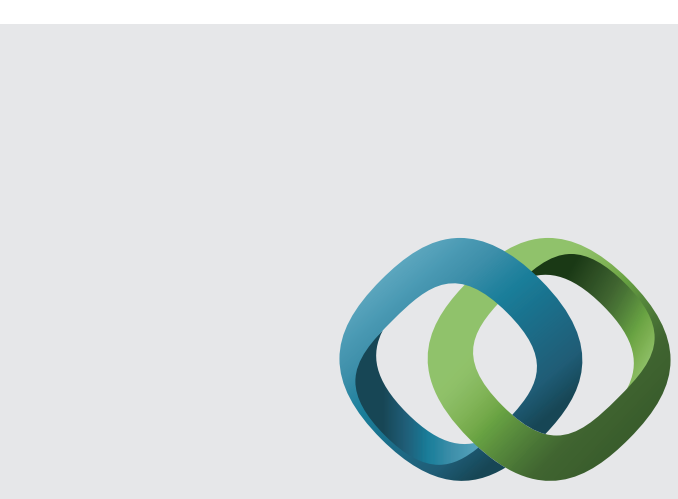

\section{Hindawi}

Submit your manuscripts at

http://www.hindawi.com
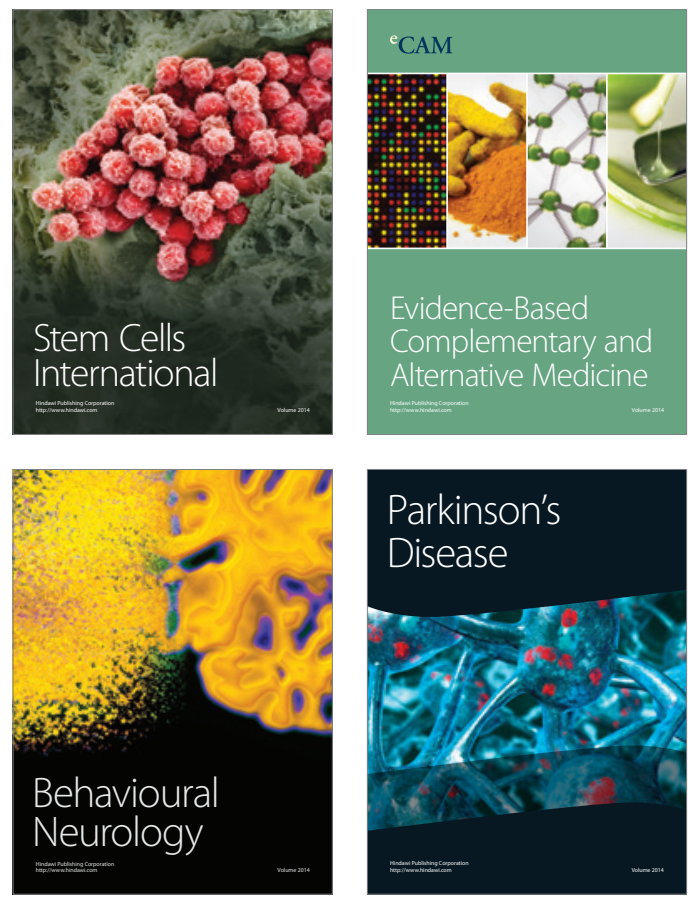
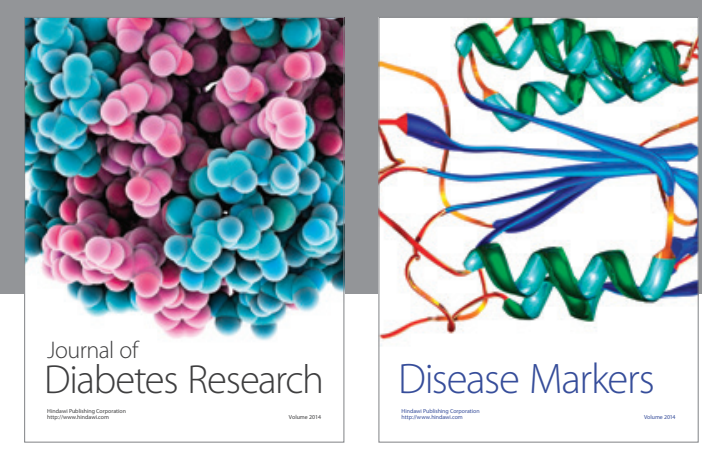

Disease Markers
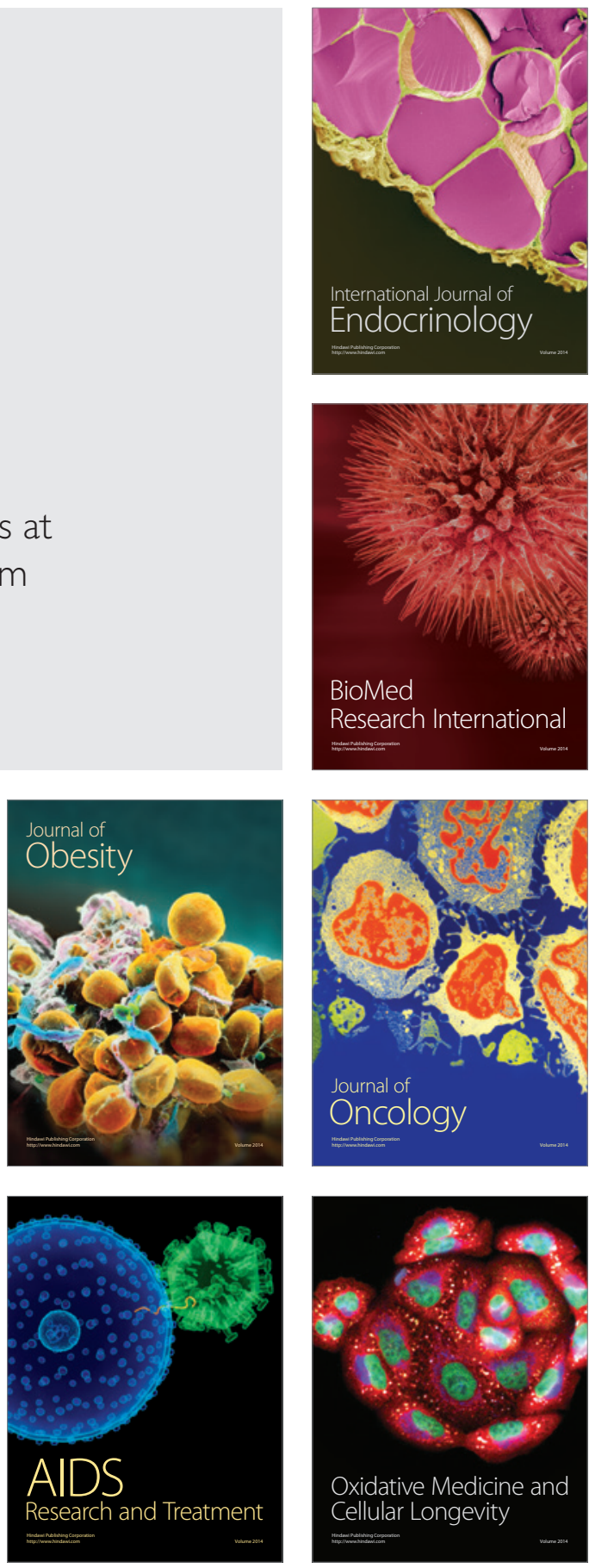